\title{
On Tight Immersions of Maximal Codimension
}

\author{
John A. Little (Ann Arbor) ${ }^{\star}$ and William F. Pohl (Minneapolis) $)^{\star}$
}

\section{Introduction}

Let $f: M^{n} \rightarrow E^{m}$ be an immersion of a compact differentiable manifold of dimension $n$ into a Euclidean space of dimension $m$. The immersion $f$ is called tight if there exists no immersion of $M^{n}$ with smaller total Lipschitz-Killing curvature [3] in any Euclidean space. The immersion $f$ is called substantial if $f\left(M^{n}\right)$ is not contained in any hyperplane of $E^{m}$. Kuiper [4] has shown that if $f$ is both tight and substantial, then $m \leqq N=\frac{1}{2} n(n+3)$. In case $m=N$ and $n=2$ (so that $N=5$ ), he has shown that if $f$ is tight and substantial then $M^{2}$ must be diffeomorphic to the real projective plane and $f$ must be an embedding onto a real algebraic variety, in fact onto a Veronese surface. In this paper we prove the corresponding result in higher dimensions.

Our hypothesis is, in fact, weaker. The immersion $f$ is said to have the two-piece property if every hyperplane divides it into at most two pieces, or more exactly, if for every hyperplane $H \subset E^{m}, f^{-1}\left(H_{1}\right)$ and $f^{-1}\left(H_{2}\right)$ are both connected sets, where $H_{1}$ and $H_{2}$ are the two open half-spaces which make up the complement of $H$ in $E^{m}$. A tight immersion has the two-piece property, but not necessarily conversely (cf. $[6,9]$ ). However, for the case of curves and surfaces the two properties are equivalent.

Let $A$ be a real vector space of dimension $n+1$ and consider the map $v \mapsto v \otimes v$ from $A$ to $A \otimes A$. Take a metric in $A$ and restrict the map to the unit sphere centered at the origin. Since $(-v) \otimes(-v)=v \otimes v$, this map takes each pair of antipodal points to the same point. Hence it induces a map of the real projective $n$-space into $A \otimes A$. As we shall see, this last map is an embedding, and the image $V$ lies substantially in an affine subspace of dimension $N=\frac{1}{2} n(n+3)$. We call any submanifold projectively equivalent to $V$ and lying in an affine or projective space a Veronese n-manifold. Any Veronese manifold is tightly embedded [4]. We can now state our main result.

* This author's work was done during tenure of a Leverhulme Fellowship at the University of Liverpool.

$\star \star$ This author's work was supported by the N.S.F. under Grant GP-20871, and by the Netherlands Organization for the Advancement of Pure Research (Z.W.O.).

13 Inventiones math, Vol 13 
Theorem 1. Let $M^{n}$ be compact, $n>1$, and $f: M^{n} \rightarrow E^{N}, N=\frac{1}{2} n(n+3)$, an immersion which is differentiable of class $C^{4}$, substantial, and which has the two-piece property. Then $M^{n}$ is diffeomorphic to a real projective $n$-space, $f$ is an embedding, and $f\left(M^{n}\right)$ is a Veronese n-manifold.

Our proof of Theorem I depends on our main local result, Theorem II, the proof of which depends in turn on Theorem III. These last two theorems, which we will state in due course, generalize theorems of Segre characterizing the Veronese surface $[10,11]$. A number of other characterizations of Veronese manifolds follow from Theorems II and III. For further information we refer the reader to [2] and [8].

We have tried to make the present paper essentially self-contained. For further information on the rich literature on tight immersions, most of it the work of Kuiper, we refer to [5] and [6]. The importance of the two-piece property seems first to have been realized by Banchoff [1].

The results of this paper were discovered and proved by the firstnamed author, who circulated a preprint "On smooth convex maps in the top dimension". Later, under the stimulation of Kuiper, both authors working together discovered the more geometric proof of Theorem II presented here. We now know a number of different proofs of these theorems, which we discuss in $\S 7$.

\section{The Veronese Manifold and the Curvature Indicatrix}

Let $A$ be as in the last $\S$ and let $g: A \rightarrow A \otimes A$ be the mapping $g(v)=$ $v \otimes v$. Let $g^{\prime}$ denote the restriction of $g$ to the unit sphere centered at the origin, $S^{n}$. The image of the map $g$, and therefore the Veronese manifold $V$ defined by $g$, lies in the space of symmetric tensors in $A \otimes A$. If we take an orthonormal basis of $A, e_{1}, \ldots, e_{n+1}$, and let

$$
e_{i} \bigcirc e_{j}=\frac{1}{2}\left(e_{i} \otimes e_{j}+e_{j} \otimes e_{i}\right),
$$

we can write the map $g$ as

$$
g\left(\sum x_{i} e_{i}\right)=\sum_{i=1}^{n+1} x_{i}^{2} e_{i} \bigcirc e_{i}+2 \sum_{1 \leqq j<k \leqq n+1} x_{j} x_{k} e_{j} \bigcirc e_{k},
$$

so that by taking the $e_{j} \bigcirc e_{k}$ as a basis for the space of symmetric tensors, we can write down the parametrization of the Veronese manifold as

$$
\left(x_{1}, \ldots, x_{n+1}\right) \rightarrow\left(x_{1}^{2}, \ldots, x_{n+1}^{2}, 2 x_{1} x_{2}, \ldots, 2 x_{1} x_{n+1}, 2 x_{2} x_{3}, \ldots, 2 x_{n} x_{n+1}\right) \text {, }
$$

where the $x_{i}$ 's are subject to the relation

$$
x_{1}^{2}+\cdots+x_{n+1}^{2}=1,
$$


showing that $V$ lies in a hyperplane in the space of symmetric tensors. It is useful to have a parametrization of a Veronese manifold in homogeneous coordinates. In fact, regarding $x_{1}, \ldots, x_{n+1}$ as homogeneous coordinates in the real projective $n$-space $P^{n}$, we get such a parametrization from (2.2), in terms of the homogeneous coordinates of $P^{N}, N=$ $\frac{1}{2} n(n+3)$, where now we ignore (2.3). (Note that $N$ is one less than the dimension of the space of symmetric tensors.) Call this map $g^{\prime \prime}: P^{n} \rightarrow P^{N}$.

This homogeneous representation has several easy consequences. First, since the quadratic monomials in (2.2) satisfy no linear relation, the Veronese manifold lies substantially in $P^{N}$. Secondly, the Veronese manifold must have the two-piece property. For, the intersection of this Veronese manifold with a hyperplane in $P^{N}$ is, by (2.2), a hyperquadric of $P^{n}$. But the complement of a hyperquadric in $P^{n}$ has at most two connected components, as may be seen in the following way. Given three points in the complement, pass a plane through them. This plane meets the hyperquadric in a conic. Two of these points may certainly be joined by a curve in this plane which does not meet the conic.

Another consequence of the homogeneous representation is that a Veronese manifold is equivariantly embedded. We explain this as follows. If $R: A \rightarrow A$ is a linear transformation, then there is an induced linear transformation $R \otimes R: A \otimes A \rightarrow A \otimes A$ such that $R \otimes R(v \otimes w)=R(v) \otimes R(w)$. $g$ is equivariant with respect to these maps, which is to say that $g \circ R=$ $(R \otimes R) \circ g$. Since the linear transformations of $A$ give the projective transformations of $P^{n}$, this implies that given any projective transformation $R^{\prime}$ of $P^{n}$, there is a projective transformation $R^{\prime \prime}$ of $P^{N}$ such that $g^{\prime \prime} \circ R^{\prime}=R^{\prime \prime} \circ g^{\prime \prime}$. In particular $R^{\prime \prime}$ takes the image of $g^{\prime \prime}$ onto itself. Using the equivariance it is easy to show that $g^{\prime \prime}$ is an embedding. For suppose $g^{\prime}(v)=\lambda g^{\prime}(w)$. We may assume that $v=e_{1}$. Then if $w=\sum x_{i} e_{i}$ it follows that

$$
e_{1} \otimes e_{1}=g^{\prime}(v)=\lambda g^{\prime}(w)=\lambda \sum x_{i} x_{j} e_{i} \otimes e_{j},
$$

from which we conclude that $x_{i}=0, i>1$, so that $v \equiv w$. Hence $g^{\prime \prime}$ is oneto-one. Now by the equivariance, $g^{\prime \prime}$ must have constant rank, which can only be $n$. The equivariant embedding property implies, finally, that given points $p, q \in P^{n}$ and a frame in the tangent space at either point, there exists a projective transformation of $P^{N}$ taking the Veronese manifold $g^{\prime \prime}\left(P^{n}\right)$ onto itself, taking $p$ to $q$, and taking the given frame at $p$ to the given frame at $q$.

The Veronese manifold enters into general differential-geometric considerations in the following way. Let $X: M^{n} \rightarrow E^{m}$, be any immersion of class $C^{2}$ of a differentiable manifold. Let $p \in M^{n}$, and $u_{1}, \ldots, u_{n}$ local coordinates valid in a neighborhood of $p$, with $u_{1}=\cdots=u_{n}=0$ at $p$. Let

$$
X_{i}=\frac{\partial X}{\partial u_{i}}, \quad X_{i j}=\frac{\partial^{2} X}{\partial u_{i} \partial u_{j}},
$$


the derivatives being evaluated at $p$. By the osculating space of $X$ at $p$ we mean the linear space through $p$ spanned by the $X_{i}$ and $X_{i j}$. If the osculating space at $p$ has dimension $N=\frac{1}{2} n(n+3)$, the maximum possible, we say that $X$ is non-degenerate at $p$. It is not hard to see that these notions are independent of the choice of the local coordinates $u_{1}, \ldots, u_{n}$, and that they are of a projective nature in the geometry of the ambient space.

Let $T_{p}$ and $N_{p}$ denote respectively the tangent and normal spaces of $X$ at $p$. For any vector $V$ in $E^{m}$ let $V^{\perp}$ denote the orthogonal projection of $V$ into $N_{p}$. Now let $x(t)$ be a curve on $M^{n}$ such that $x(0)=p$. Then the orthogonal projection into $N_{p}$ of the second derivative of $X(x(t))$ at 0 depends only on the first derivative of $X(x(t))$ at 0 , as is well known (at least in the case of curves on surfaces in ordinary space, and as is proved in the same way in higher dimensions). Thus we have a map $\mathscr{N}: T_{p} \rightarrow N_{p}$ which assigns to each $v \in T_{p}$ the orthogonal projection in $N_{p}$ of the second derivative vector of a curve on $M^{n}$ through $p$ whose tangent vector at $p$ is $v$. To find an analytic expression for $\mathcal{N}$ we consider the curves

$$
u_{i}=x_{i} t, \quad 1 \leqq i \leqq n .
$$

Then $X^{\prime}(0)=\sum x_{i} X_{i}$, and $X^{\prime \prime}(0)^{\perp}=\sum_{i, j=1}^{n} x_{i} x_{j} X_{i j}^{\perp}$. Hence

$$
\mathscr{N}\left(\sum x_{i} X_{i}\right)=\sum_{i, j=1}^{n} x_{i} x_{j} X_{i j}^{\perp}
$$

Now assume that $X$ is non-degenerate at $p$. It follows that $X_{i j}^{\perp}, i \leqq j$, are linearly independent, and we may identify $X_{i j}^{\perp}$ with $X_{i} \bigcirc X_{j}$. Thus $\mathcal{N}$ is identified with the map $g$ such that $g(v)=v \otimes v$. Letting $S_{p}$ denote the sphere of tangent vectors of unit length to $X$ at $p$, we find that $\mathscr{N}\left(S_{p}\right)$ is a Veronese $(n-1)$-manifold, which we call the curvature indicatrix. It lies in a hyperplane in $N_{p}$ (coming from the condition $x_{1}^{2}+\cdots+x_{n}^{2}=1$ ), which we call the indicatrix plane, $I_{p}$. The mapping $\mathscr{N}$ is two-to-one (except at 0 ), identifying $v$ and $-v$, and the image $\mathcal{N}\left(T_{p}\right)$ is a half cone consisting of rays from the origin through the points of the curvature indicatrix. We call this cone the curvature indicatrix cone. The indicatrix plane, $I_{p}$, does not contain the origin, so there is no immersed curve on $M^{n}$ with zero curvature at $p$. Since $I_{p} \subset N_{p}, I_{p}$ and $T_{p}$ are in general position and their linear span is all of $E^{N}$.

A Veronese manifold is itself everywhere non-degenerate. To prove this it suffices to check it at any point $\left(x_{1}=1, x_{2}=\cdots=x_{n+1}=0\right.$ is convenient), since the Veronese manifold is equivariantly embedded and non-degeneracy is a projective notion. But we can avoid calculation by the following geometric argument.

Let $U$ be a linear subspace of $P^{n}$ of dimension $r$; then its image under $g^{\prime \prime}$ is a Veronese $r$-manifold, as can be seen from (2.2) by taking homo- 
geneous coordinates $x_{1}, \ldots, x_{n+1}$ in $P^{n}$ such that $U$ is defined by $x_{r+2}=\cdots=x_{n+1}=0$. Let $V=g^{\prime \prime}\left(P^{n}\right)$. We call $g^{\prime \prime}(U)$ a Veronese submanifold of $V$. Now a Veronese manifold of dimension one is just a non-singular conic. Since any two points of $P^{n}$ may be joined by a line, any two points of a Veronese manifold may be joined by a conic lying in the Veronese manifold. This implies that $V$ is non-degenerate; for if the osculating space at $p \in V$ were contained in a hyperplane $H$, then $H$ would contain $T_{p}$ and. $\mathcal{F}\left(T_{p}\right)$, by (2.4), and hence the tangent and curvature vectors at $p$ of all curves on $V$ through $p$, and hence the planes of the one-dimensional Veronese submanifolds passing through $p$, and hence $V$ itself. But this contradicts the already proven fact that $V$ lies substantially in $P^{N}$.

In terms of the Euclidean representation (2.2), (2.3), a Veronese submanifold is defined in the following fashion. Take a linear subspace through the origin of $A$ and intersect with the unit sphere $S^{n}$. The image of this intersection under the map $\mathrm{g}^{\prime}$ is a Veronese submanifold.

Let $V^{n-1} \subset V^{n} \subset P^{N}$ be an $(n-1)$-dimensional Veronese submanifold of a Veronese $n$-manifold. We claim that there is a unique hyperplane $J$ of $P^{N}$ such that $J \cap V^{n}=V^{n-1}$ and such that $J$ is tangent to $V^{n}$ at each point of $V^{n-1}$, which is to say that $J$ contains the tangent space to $V^{n}$ at each point of $V^{n-1}$. To show this it suffices to take $V^{n-1}$ to be defined by $x_{n+1}=0$, using our usual homogeneous coordinates, since $V^{n}$ is equivariantly embedded. Referring to (2.2) we see that $V^{n-1}$ then lies in the hyperplane $J$ of $P^{N}$ defined by setting the $(n+1)$-st coordinate of $P^{N}$ equal to zero, and any point of $V^{n}$ for which this coordinate is zero must lie in $V^{n-1}$. This coordinate is $x_{n+1}^{2}$, on $V^{n}$, so that $V^{n}$ lies locally on one side of $J$. This implies that $J$ is tangent to $V^{n}$ along $V^{n-1}$, as required. This last determines $J$ uniquely. For, referring to (2.1) for purposes of notation, we see by differentiating that the tangent space to $g$ at $e_{i}$ is spanned by $e_{i} \bigcirc e_{1}, \ldots, e_{i} \bigcirc e_{n+1}$. Since $J$ contains $g^{\prime \prime}\left(e_{1}\right), \ldots, g^{\prime \prime}\left(e_{n}\right)$ it must contain all $e_{i} \bigcirc e_{j}, 1 \leqq i \leqq n, 1 \leqq j \leqq n+1$. But these are sufficient to span $J$. Our claim is now established. We call $J$ an e.s. hyperplane of $V^{n}$ ("e.s." standing for "extremal support").

Let us note here that $\boldsymbol{J}$ must contain the curvature vectors of all curves on $V^{n-1}$. (We assume that $V \subset E^{N}$ for a moment.) Since $J$ contains the tangent spaces to $V^{n}$ at the points of $V^{n-1}$, it must contain $\mathscr{N}(v)$ for every $v$ tangent to $V^{n-1}$, where $\mathcal{N}$ is the curvature indicatrix cone for $V^{n}$.

Consider now an $r$-dimensional Veronese manifold $W$ which happens to be contained in $V^{n}$. We claim that $W$ is a Veronese submanifold of $V^{n}$ in the sense defined above. For, choose a point $p$ on $W$ and let $V^{n-1}$ be a Veronese submanifold of $V^{n}$, passing through $p$, whose tangent space at $p$ contains the tangent space to $W$ at $p$. Now $V^{n-1}$ is contained in an e.s. hyperplane $J$. If we have any conic lying on $W$ and passing through $p$ its tangent vector $v$ and its curvature vector must be contained in $J$, 
since $J$ contains $T_{p}$ and $\mathscr{N}(v)$. It follows that $J$ contains the conic. And since any point of $W$ may be joined to $p$ by such a conic, $J \supset W$. But since $J \cap V^{n}=V^{n-1}$, we must have $W \subset V^{n-1}$. We can repeat this argument until we have a sequence of Veronese submanifolds

$$
V^{\prime} \subset V^{r+1} \subset \cdots \subset V^{n-1} \subset V^{n}
$$

with $W=V^{\prime \prime}$, which establishes the claim.

The other properties of Veronese manifolds which we need to prove could be established at this point by algebraic arguments. But it is more convenient to derive them as special cases of more general results which we prove later.

\section{Consequences of the Two-Piece Property}

Throughout this $\S M^{n}$ will denote a compact differentiable manifold of dimension $n$, and $f: M^{n} \rightarrow E^{m}$ a $C^{2}$ immersion having the two-piece property. It is easy to see that this implies that $M^{n}$ is connected.

If $X \subset E^{m}$ is some subset, we say that a hyperplane $H \subset E^{m}$ is a hyperplane of support of $X$ if $X$ lies in one of the two closed half-spaces of $E^{m}$ determined by $H$ and if $X \cap H \neq 0$. Note that if $X$ is an immersed submanifold then $H$ must contain the tangent space of $X$ at each point of $X \cap H$. A hyperplane containing the tangent space at a point of an immersed submanifold is said to be tangent at that point. A smooth real-valued function $F$ on $M^{n}$ is said to have a critical point at $p \in M^{n}$ if $d F=0$ at $p$, and a critical point $p$ of $F$ is said to be non-degenerate if the Hessian, $d^{2} F$, is non-degenerate at $p$. (Let us not confuse this notion with that of a non-degenerate immersion, defined in the last $\S$.) If $v$ is a vector in $E^{m}$, the inner product $v \cdot f: M^{n} \rightarrow R$ is called a height function on $M^{n}$.

Proposition 3.1. a) Let $H$ be a hyperplane of $E^{m}, p \in M^{n}$, and $U$ a neighborhood of $p$ such that $H$ supports $f(U)$ and $f(U) \cap H=f(p)$. Then $H$ supports $f\left(M^{n}\right)$.

b) Any non-degenerate local maximum of a height function on $M^{n}$ is a global maximum.

Proof. a) By making $U$ smaller, if necessary, we can arrange that $H$ does not meet $f(\partial U)$, where $\partial U$ is the boundary of $U$. Let $\varepsilon$ denote the distance in $E^{m}$ from $f(\partial U)$ to $H$. Since $M^{n}$, and hence $\partial U$, are compact, $\varepsilon>0$. If $H$ were not a support hyperplane of $f\left(M^{n}\right)$ we could move $H$ parallelly toward $f(\hat{\partial} U)$ a distance of $\frac{1}{2} \varepsilon$ to obtain a hyperplane cutting $M^{n}$ into at least three pieces, which would contradict the two-piece property. Hence $H$ must support $f\left(M^{n}\right)$.

b) Let $v \cdot f$ be a height function with a non-degenerate local maximum at $p \in M^{n}$. Then, by Morse's lemma, we can find local coordinates $x_{1}, \ldots, x_{n}$, 
valid in a neighborhood $U$ of $p$, such that $v \cdot f=v \cdot f(0)-x_{1}^{2}-x_{2}^{2}-\cdots-x_{n}^{2}$ in $U$. The hyperplane $H$ through $f(p)$ perpendicular to $v$ supports $f(U)$, and $f(U) \cap H=p$. Hence by Part a) $H$ is a support plane of $f\left(M^{n}\right)$, which implies that $v \cdot f$ has a global maximum at $p$. This completes the proof.

We call a point $p \in M^{n}$ an extreme point of $f$ if there exists a height function with a non-degenerate local maximum at $p$ (and hence a global maximum at $p$ ).

Proposition 3.2. a) The set of extreme points of $f$ is open and non-empty.

b) Every extreme point is simple; i.e. if $p$ is an extreme point and $f(p)=f(q)$, then $p=q$.

Proof a) Let $\pi: N \rightarrow M^{n}$ be the full normal bundle of $f$, and let $g: N \rightarrow E^{m}$ be the "Gauss map", i.e. the map which takes each normal vector to the same vector bound at the origin of $E^{m}$. It is well known that the critical points of a height function $v \cdot f$ are just the points $\pi\left(g^{-1}(v)\right)$, and that if $v^{\prime} \in g^{-1}(v)$ and $p=\pi\left(v^{\prime}\right)$, then $v \cdot f$ has a non-degenerate critical point at $p$ if and only if $g$ has maximal Jacobian rank at $v^{\prime}$. Now by Sard's theorem, the image under $g$ of the critical locus of $g$ has measure zero in $E^{m}$. But for every vector $v$ in $E^{m}$ the height function $v \cdot f$ attains its maximum value, since $M^{n}$ is compact. Consequentiy there must be an abundance of height functions taking non-degenerate maxima, and hence an abundance of extreme points.

To show that the set of extreme points is open, suppose that $p$ is an extreme point and let $v$ be a vector such that $v \cdot f$ has a non-degenerate maximum at $p$. Then, regarding $v$ as lying in $N_{p}, g$ must have maximal rank at $v$, and hence must have maximal rank in some connected neighborhood $U$ of $v$ in $N$. The determinant of the Hessian matrix of $v^{\prime} \cdot f$ cannot vanish at $\pi\left(v^{\prime}\right)$ for $v^{\prime} \in U$; hence for each $v^{\prime}$ in $U v^{\prime} \cdot f$ has a non-degenerate maximum at $\pi\left(v^{\prime}\right)$. Hence $\pi(U)$ is a neighborhood of $p$ consisting of extreme points.

b) Suppose $p \in M^{n}$ is an extreme point of $f$ and that $f(p)=f(q)$, $p \neq q$. Let $v$ be a vector such that $v \cdot f$ has a non-degenerate maximum at $p$, and let $H$ be the hyperplane of $M^{n}$ through $f(p)$ and perpendicular to $v$. By Morse's lemma, we can find a closed neighborhood $U$ of $p$ in $M^{n}$ such that $H$ supports $f(U)$ and $f^{-1}(H) \cap U=p$. Let $\varepsilon$ denote the distance, in $E^{m}$, from $H$ to $f(\partial U)$. Let $H^{\prime}$ be a hyperplane parallel to $H$ at a distance $\frac{1}{2} \varepsilon$ toward $f(\partial U)$. Then $H^{\prime}$ divides $M^{n}$ into at least three components, one containing $p$, one containing $q$, and one containing $\partial U$. Hence the assumption that $p \neq q$ is false, which completes the proof.

Proposition 3.3. Let $p \in M^{n}$ be an extreme point and let $H$ be a hyperplane in $E^{m}$ tangent to $f$ at $p$. Then $H$ supports $f\left(M^{n}\right)$ if and only if $\mathcal{N}\left(S_{p}\right)$ lies in one of the closed half-spaces determined by $\mathrm{H}$. 
Proof. Let $v$ be a vector perpendicular to $H$. Then $v$ is normal to $f$ at $p$, so that the function $v \cdot f$ has a critical point at $p$. Let $x(t)$ be an immersed curve on $M^{n}$ such that $x(0)=p$ and let $w$ denote the tangent vector to $x$ at 0 . Then

$$
\frac{d^{2} v \cdot(f \circ x)}{d t^{2}}(0)=v \cdot \frac{d^{2}(f \circ x)}{d t^{2}}(0)=v \cdot \mathscr{N}(w) .
$$

Now if $\mathscr{N}\left(S_{p}\right)$ lies on both sides of $H$, then (3.1) will be positive for some curves on $M^{n}$ through $p$ and negative for others. Hence $v \cdot f$ will have a non-degenerate local minimum along some curves at $p$ and a local maximum along others. Hence $H$ is not a support hyperplane of $f\left(M^{n}\right)$, and the Proposition is proved in one direction. Note that we have not yet used the two-piece property.

If $H$ does not meet $\mathscr{N}\left(S_{p}\right)$, which implies that $\mathscr{N}\left(S_{p}\right)$ lies on one side of $H$, since $\mathcal{N}\left(S_{p}\right)$ is connected, (3.1) will have the same sign, no matter what curve $x(t)$ we take. Hence the Hessian of $v \cdot f$ is definite. Assume that $v$ points into the half space determined by $H$ and not containing $\mathscr{N}\left(S_{p}\right)$. Then $v \cdot f$ takes a non-degenerate local maximum at $p$. Hence by Proposition $3.1 \mathrm{~b}$ ) it takes a global maximum at $p$, which implies that $H$ supports $f\left(M^{n}\right)$.

Finally, suppose $H$ meets and supports $\mathscr{N}\left(S_{p}\right)$. We may assume that $v$ points into the half-space not containing $\mathscr{N}\left(S_{p}\right)$. Since $p$ is an extreme point there exists a vector $v^{\prime}$ such that $v^{\prime} \cdot f$ has a non-degenerate local maximum at $p$, and hence a global maximum at $p$. Hence the hyperplane $H^{\prime}$ through $f(p)$ and perpendicular to $v^{\prime}$ supports $f\left(M^{n}\right)$. Now $H^{\prime}$ cannot cut $\mathscr{N}\left(S_{p}\right)$, by the first paragraph of this proof. Hence $\mathcal{N}\left(S_{p}\right)$ lies in the intersection of two closed half-spaces, one determined by $H$ and the other by $H^{\prime}$. Now turn $H^{\prime}$ about $H^{\prime} \cap H$ till it reaches $H$, so that all the intermediate hyperplanes do not meet $\mathscr{N}\left(S_{p}\right)$. By the last paragraph, these all support $f\left(M^{n}\right)$. Hence so does the limiting and final one, namely $H$. (To understand these last arguments, project everything orthogonally into the plane spanned by $v$ and $v^{\prime}$.) This concludes the proof.

Corollary 3.4. Assume that $f$ is differentiable of class $C^{3}$. Then any hyperplane which supports $f\left(M^{n}\right)$ to the second-order supports $f\left(M^{n}\right)$ to the third order. By this we mean that if $H$ is a hyperplane in $E^{m}$ tangent to $f$ at $p$ which supports $\mathscr{N}\left(T_{p}\right)$, and if $X(t)=f(x(t))$ is any curve on $M^{n}$ such that $x(0)=p$ and $X^{\prime \prime}(0)$ lies in $H$, then $X^{\prime \prime \prime}(0)$ lies in $H$.

Proof. Suppose there is such a curve with $X^{\prime \prime \prime}(0)$ not in $H$. Expand $X(t)$ as a finite Taylor's series:

$$
X(t)=X(0)+t X^{\prime}(0)+\frac{1}{2} t^{2} X^{\prime \prime}(0)+\frac{1}{6} t^{3}\left(X^{\prime \prime \prime}(0)+t R(t)\right),
$$


where $R(t)$ is continuous in $t$. If $v$ is a non-zero vector in $E^{m}$ normal to $H$, then

$$
v \cdot(X(t)-X(0))=\frac{1}{6} t^{3}\left(v \cdot X^{\prime \prime \prime}(0)+t v \cdot R(t)\right),
$$

which takes both positive and negative values near zero, so that $H$ does not support $f\left(M^{n}\right)$. But this contradicts Proposition 3.3. Hence the corollary.

Proposition 3.5. Let $p \in M^{n}$ be an extreme point. Then $f\left(M^{n}\right)$ is contained in the osculating space to $f$ at $p$.

Proof. If the osculating space to $f$ at $p$ is all of $E^{m}$, there is nothing to prove. Suppose then that the osculating space to $f$ at $p$ is a linear space $J$ of dimension $r<m$. Since $p$ is an extreme point, there is a vector $v$ in $E^{m}$ such that $v \cdot f$ has a non-degenerate maximum at $p$; let $H$ be the hyperplane through $f(p)$ perpendicular to $v$. Now $H$ does not meet $\mathscr{N}\left(S_{p}\right)$; otherwise there would be a curve on $M^{n}$ through $p$ such that the second derivative of $v \cdot f$ along it would be zero, as we see from (3.1); but this contradicts the fact that the Hessian of $v \cdot f$ at $p$ is negative definite. Since $J$ contains $\mathscr{N}\left(S_{p}\right)$ by (2.4), it follows that $H$ does not contain $J$, so that $H \cap J$ is a linear subspace of dimension $r-1$; call it $K$. Since $\mathscr{N}\left(S_{p}\right)$ is connected and does not meet $K$, it must lie in one if the half-spaces of $J$ determined by $K$, call it $J^{+}$. Hence any hyperplane of $E^{m}$ containing $K$ but not $\boldsymbol{J}$ supports $\mathscr{N}\left(T_{p}\right)$, and hence, by Proposition 3.3, supports $f\left(M^{n}\right)$. It follows that $f\left(M^{n}\right)$ lies in the intersection of all closed halfspaces containing $J^{+}$determined by hyperplanes cutting $J$ in $K$. But this intersection is just $J^{+}$. It follows that $f\left(M^{n}\right)$ lies in $J$.

Corollary 3.6. If $f: M^{n} \rightarrow E^{m}$ is substantial, then $m \leqq N=\frac{1}{2} n(n+3)$.

Proof. The osculating space at $p$ has dimension $\leqq N$.

Corollary 3.7. If $f: M^{n} \rightarrow E^{N}$ is substantial, then $f$ is non-degenerate at every extreme point.

Proposition 3.8. Let $f: M^{n} \rightarrow E^{N}$. Then every non-degenerate point is an extreme point.

Proof. Let $f$ be non-degenerate at $p$ and let $H$ be a hyperplane containing the tangent plane $T_{p}$ and parallel to the indicatrix plane $I_{p}$. Let $v$ be a non-zero vector perpendicular to $H$ and pointing into the halfspace determined by $H$ and not containing $I_{p}$. Then $v \cdot f$ has a nondegenerate maximum at $p$, as we see from $(3.1)$.

\section{The Dual Manifold}

The results of this $\S$ are of a local nature. Let $M^{n}$ be an arbitrary connected differentiable manifold of dimension $n$, and let $f: M^{n} \rightarrow E^{N}$, $N=\frac{1}{2} n(n+3)$, be an immersion which is differentiable of class $C^{3}$, non- 
degenerate, and which has the property that any hyperplane which supports $f\left(M^{n}\right)$ to the second order supports $f\left(M^{n}\right)$ to the third order. By this we mean, as we said before, that if $H$ is any hyperplane tangent to $f$ at $p$ which supports $\mathscr{N}\left(T_{p}\right)$, and if $x(t)$ is any curve on $M^{n}$ such that $x(0)=p$ and $X^{\prime \prime}(0)$ lies in $H$, then $X^{\prime \prime \prime}(0)$ lies in $H$. By Corollary $3.4, f$ has this property if it has the two-piece property. Veronese $n$-manifolds must therefore have this property, since they have the two-piece property. We now state the main local result of this paper. The first part of the proof will be given in this $\S$ and the completion in $\S 6$.

Theorem II. Let $n \geqq 2$ and suppose $f: M^{n} \rightarrow E^{N}$ is differentiable of class $C^{4}$, non-degenerate, and has the property that any hyperplane which supports $f\left(M^{n}\right)$ to the second order supports $f(M)$ to the third order. Then $f\left(M^{n}\right)$ is contained in a Veronese $n$-manifold.

Remarks. The condition that any hyperplane which supports to the second order supports to the third is equivalent to the condition that "the characteristic curves are degenerate" [7]. Later on, following Proposition 4.9, we will put this condition in another form. Theorem II was proved by Segre $[10,11]$ for $n=2$, and from it follow many of the classical characterizations of the Veronese surface.

Let us consider a non-degenerate immersion $g: M^{n} \rightarrow E^{N}$, without assuming any other special properties. Let $p \in M^{n}$, and let $H^{\prime}$ be an e.s. hyperplane of $\mathscr{N}\left(S_{p}\right)$ in the indicatrix plane $I_{p}$. Since $I_{p}$ and $T_{p}$ are in general position, $H^{\prime}$ and $T_{p}$ together span a hyperplane $H$, which we call an e.s. hyperplane of $g$ at $p$. We claim that this agrees with the previous definition in case $g$ is a Veronese manifold, at least for $n>1$. For if $V \subset E^{N}$ is a Veronese manifold and $H$ an e.s. hyperplane of $V$ in the sense of $\S 2$, with $p \in V \cap H$, then $H$ supports $V$ locally and hence globally, since $V$ is connected. Therefore, by Proposition 3.3,H supports $\mathscr{N}\left(T_{p}\right)$. Now $V^{n-1}=V \cap H$ is a Veronese submanifold and hence $H$ contains the curvature vectors of all curves on $V^{n-1}$. Since $H$ contains $T_{p}$ it must therefore contain $\mathscr{N}\left(S_{p}^{\prime}\right)$, where $S_{p}^{\prime} \subset S_{p}$ is the set of unit vectors at $p$ tangent to $V^{n-1}$. It follows that $H \cap I_{p}$ supports $\mathscr{A}\left(S_{p}\right)$ and contains the Veronese submanifold $\mathscr{N}\left(S_{p}^{\prime}\right)$, so that $H \cap I_{p}$ is an e. s. hyperplane of $\mathcal{T}\left(S_{p}\right)$ in $I_{p}$, from which it follows that $H$ is an e.s. hyperplane in the sense of this $\S$. On the other hand, if $H$ is an e.s. hyperplane of $V$ at $p$ in the sense of this $\S$, it must contain $T_{p}$ and the Veronese submanifold $\mathscr{N}\left(S_{p}^{\prime}\right)$, for some great $(n-2)$-sphere $S_{p}^{\prime}$ of $S_{n}$. If $V^{n-1}$ is a Veronese submanifold of $V$ through $p$ whose tangent space contains $S_{p}^{\prime}$, then $H^{\prime}$ must contain the tangent and curvature vectors at $p$ of all curves on $V^{\boldsymbol{n}-1}$ through $p$, in particular those of the conics on $V^{n-1}$ through $p$. Hence $H$ contains all such conics, and since each point of $V^{n-1}$ may be joined to $p$ by such a conic, $H$ contains $V^{n-1}$. Since $H$ supports $V$, by Propo- 
sition 3.3 (applicable in this case because $V$ has the two-piece property) $H$ must be an e.s. hyperplane of $V$ in the sense of $\S 2$. This establishes the claim.

An e.s. hyperplane at $p \in M^{n}$ is determined by giving a Veronese submanifold of dimension $n-2$ of $\mathscr{N}\left(S_{p}\right)$. Such a Veronese submanifold is determined by giving a great $(n-2)$-sphere of $S_{p}$. This in turn may be determined by giving an (n-1)-plane through the origin of $T_{p}$. Note that an e.s. hyperplane $H$ supports $\mathscr{N}\left(S_{p}\right)$ and contains $g(p)$, from which it follows that $H$ supports the curvature cone $\mathscr{N}\left(T_{p}\right)$. If $h$ is an $(n-1)$-plane through the origin of $T_{p}$ such that $\mathscr{N}\left(h \cap S_{p}\right)=\mathscr{N}\left(S_{p}\right) \cap H$, then $H$ must be tangent to $\mathscr{N}\left(T_{p}\right)$ along $\mathscr{N}(h)$.

Lemma 4.1. Let $p \in M^{n}, U$ a neighborhood of $p$ in $M^{n}$, and $H$ a hyperplane of $E^{N}$ such that $g^{-1}(H) \cap U$ is an embedded submanifold of codimension one. Suppose that $H$ contains $T_{p}$, and that $H$ supports $\mathcal{N}\left(T_{p}\right)$ or that $H$ supports $g\left(M^{n}\right)$; then $H$ is an e.s. hyperplane of $g$ at $p$.

Proof. If $H$ supports $g\left(M^{n}\right), H$ must support $\mathscr{N}\left(T_{p}\right)$, as can be seen from the proof of Proposition 3.3. Hence it suffices to assume that $H$ supports $\mathscr{N}\left(T_{n}\right)$. Now $H$ must contain all curvature vectors of curves on $g^{-1}(H) \cap U$. It follows that $H$ contains the Veronese submanifold $\mathcal{N}\left(h_{p}\right)$, where $h_{p} \subset T_{p}$ is the tangent space to $g^{-1}(H) \cap U$. Hence $H \cap I_{p}$ is an e.s. hyperplane of $\mathscr{N}\left(S_{p}\right)$ containing $\mathscr{N}\left(h_{p} \cap S_{p}\right)$, from which the lemma follows.

These preliminaries out of the way, let us consider the map $f$ again. The next considerations will lead to the definition of the dual manifold. Let $p \in M^{n}$, and $h \subset T_{p}$ an $(n-1)$-dimensional linear subspace. Let $H^{\prime}$ be the e.s. hyperplane, in $I_{p}$, of $\mathcal{N}\left(S_{p}\right)$ which contains $\mathscr{N}\left(h \cap S_{p}\right)$. Let $\varphi(h)$ be the e.s. hyperplane of $f$ at $p$ spanned by $T_{p}$ and $H^{\prime}$. Then $\varphi$ is a mapping whose domain is the bundle of linear subspaces of dimension $n-1$ of the tangent spaces of $M^{n}$. We denote this bundle by $\pi: G_{n-1} T\left(M^{n}\right) \rightarrow M^{n}$. The range of $\varphi$ is the space of hyperplanes of $E^{N}$. It is convenient to add the hyperplane at infinity to this space, obtaining thereby the dual projective space $P^{N *}$. Thus $\varphi: G_{n-1} T\left(M^{n}\right) \rightarrow P^{N *}$. The mapping $\varphi$ may also be defined in purely projective terms. In fact, $\varphi(h)$ is the unique hyperplane which supports $f$ to the second order at $p \in M^{n}$ and which contains the osculating planes at $p$ to all curves lying on $M^{n}$ tangent to $h$ at $p$.

Proposition 4.2. $\varphi$ has everywhere Jacobian rank $n$. The restriction of $\varphi$ to any fibre $G_{n-1} T_{p}$ is one-to-one and has rank $n-1$, i.e. is an embedding.

Proof. We assume $n \geqq 2$. The assertion that $\varphi$ restricted to $G_{n-1} T_{p}$ is one-to-one is essentially the assertion that each e.s. hyperplane of the Veronese manifold $\mathscr{N}\left(S_{p}\right)$ meets $\mathscr{N}\left(S_{p}\right)$ in an unique Veronese $(n-2)$ - 
dimensional submanifold. But this we know already. From this, and the equivariant embedding property of $\mathcal{N}\left(S_{p}\right)$, it follows that $\varphi$ restricted to $G_{n-1} T_{p}$ has constant rank $n-1$. We proceed, then, to show that $\varphi$ has rank $n$, and we accomplish this by representing $\varphi(h)$ as an exterior $N$-vector, differentiating $\varphi$ in $2 n-1$ independent directions, and showing that the resulting $N$-vectors, together with $\varphi(h)$, span a space of dimension $n+1$.

Let $h \in G_{n-1} T\left(M^{n}\right)$ be arbitrary and let $p=\pi(h) \in M^{n}$. Introduce local coordinates $u_{1}, \ldots, u_{n}$ on $M^{n}$ in a neighborhood of $p$ such that $h$ is spanned by $\partial / \partial u_{1}, \ldots, \partial / \partial u_{n-1}$. Let $h_{i}(t)$ denote the linear span of

$$
\frac{\partial}{\partial u_{1}}, \ldots, \frac{\partial}{\partial u_{i-1}}, \frac{\partial}{\partial u_{i}}+t \frac{\partial}{\partial u_{n}}, \ldots, \frac{\partial}{\partial u_{n-1}},
$$

and let us compute $\varphi\left(h_{i}(t)\right)$. We use the notation of $\S 2$, so we will consider $f: M^{n} \rightarrow E^{N}$ as a position-vector function, which we write as $X: M^{n} \rightarrow E^{N}$. Now

so that

$$
\mathscr{N}\left(\sum x_{j} \frac{\partial}{\partial u_{j}}\right)=\sum x_{k} x_{j} X_{k j}^{\perp}
$$

$$
\frac{\partial \mathscr{N}}{\partial x_{k}}=2 \sum x_{j} X_{k j}^{\perp}
$$

Hence the tangent space to the whole curvature cone $\mathscr{N}$ at $\partial / \partial x_{l}$ is spanned by $X_{k l}^{\perp}, 1 \leqq k \leqq n$, and the tangent space to $\mathscr{N}$ at $\partial / \partial u_{i}+t \partial / \partial u_{n}$ is spanned by $X_{k i}^{\perp}+t X_{k n}^{\perp}, 1 \leqq k \leqq n$. Hence $\varphi\left(h_{i}(t)\right)$ must contain $X_{1}, \ldots, X_{n}$, $X_{j k}(j \leqq k,(j, k) \neq(i, n),(i, i),(n, n)), X_{i i}+t X_{i n}$, and $X_{n i}+t X_{n n}$. These vectors are linearly independent, since $f$ is non-degenerate, and they are sufficient in number to span $\varphi\left(h_{i}(t)\right)$.

In order to represent $\varphi\left(h_{i}(t)\right)$ as a multivector, we introduce homogeneous coordinates in $E^{N}$, say $\xi_{0}, \ldots, \xi_{N}$, such that $y_{j}=\xi_{j} / \xi_{0}$ are ordinary coordinates in $E^{N}$. In these coordinates we may represent $f$ by the vector function

$$
Y=\left(1, y_{1} \circ f, \ldots, y_{N} \circ f\right) \text {, }
$$

and hence we may represent $\varphi\left(h_{i}(t)\right)$ as

where

$$
\varphi\left(h_{i}(t)\right)=Y \wedge Y_{1} \wedge \cdots \wedge Y_{n} \wedge \prod_{\substack{j \leq k \\(j, k) \neq(i, n),(i, i),(n, n)}} Y_{j k} \wedge\left(Y_{i i}+t Y_{i n}\right) \wedge\left(Y_{n i}+t Y_{n n}\right),
$$

$$
Y_{j}=\frac{\partial Y}{\partial u_{j}}, \quad Y_{j k}=\frac{\partial^{2} Y}{\partial u_{k} \partial u_{j}} .
$$


It is easy now to differentiate with respect to $t$. The derivative of $\varphi\left(h_{i}(t)\right)$ at $t=0$, we call $\varphi_{i}$.

$$
\varphi_{i}=\frac{d \varphi \circ h_{i}}{d t}(0)=Y \wedge Y_{1} \wedge \cdots \wedge Y_{n} \wedge \prod_{\substack{j \leq k \\(1, k) \neq(i, n),(i, i),(n, n)}} Y_{j k} \wedge Y_{i i} \wedge Y_{n n}= \pm \Omega_{i n},
$$

where by $\Omega_{j k}$ we understand the product of $Y, Y_{l}, Y_{l m}, 1 \leqq l, m \leqq n, l \leqq m$, in some order with only $Y_{j k}$ left out. Clearly $\varphi(h)=\Omega_{n n}, \varphi_{i}, 1 \leqq i \leqq n-1$, are linearly independent. The $\varphi_{i}$ are directional derivatives of $\varphi$ along a maximal independent set of fibre directions of $G_{n-1} T\left(M^{n}\right)$, which shows again that $\varphi$ restricted to $G_{n-1} T_{p}$ has constant rank $n-1$.

To differentiate $\varphi$ along the base directions, we write

Hence

$$
\varphi(h)= \pm Y \wedge Y_{1} \wedge \cdots \wedge Y_{n} \wedge \prod_{\substack{j \leq k \\(j, k) \neq(n, n)}} Y_{j k}= \pm \Omega_{n n}
$$

$$
\varphi_{u_{2}} \equiv \frac{\partial \varphi}{\partial u_{i}}(h)=Y \wedge Y_{1} \wedge \cdots \wedge Y_{n} \wedge \sum_{\substack{l \leq m \\(1, m) \neq(n, n)}} \pm Y_{i l m} \wedge \prod_{\substack{j \leq k \\(j . k) \neq(n, n) \\(j . k) \neq(l, m)}} Y_{j k}
$$

for $i<n$, and

$$
\begin{aligned}
\varphi_{u_{n}}=\frac{\partial \varphi}{\partial u_{n}}(h)= & \pm Y \wedge Y_{1} \wedge \cdots \wedge Y_{n-1} \wedge Y_{n n} \wedge \prod_{\substack{j \leq k \\
Y_{i k}}} \\
& \pm Y \wedge Y_{1} \wedge \cdots \wedge Y_{n} \wedge(\cdots) .
\end{aligned}
$$

Clearly $\varphi(h), \varphi_{1}, \ldots, \varphi_{n-1}, \varphi_{u_{n}}$ are linearly independent. We claim that $\varphi_{u_{1}}$ is a linear combination of $\varphi(h), \varphi_{1}, \ldots, \varphi_{n-1}$, for all $i<n$.

To prove this claim we recall the elementary fact that any homogeneous cubic polynomial of several variables is a linear combination of cubes of homogeneous linear polynomials. From this it follows that any homogeneous linear differential operator of order three is a linear combination of cubes of linear differential operators of order one with constant coefficients. This implies that $Y_{i l m}(p)$, where $i, l, m<n$, is a linear combination of third derivatives of $Y$ along curves on $M^{n}$ through $p$ tangent to $h$. But all such third derivatives must lie in $\varphi(h)$, by the hypothesis that a hyperplane supporting to the second order must support to the third order. Hence $Y_{i l m}(p), i, l, m<n$ is a linear combination of $Y, Y_{1}, \ldots, Y_{n}, Y_{j k},(j, k) \neq(n, n)$. Substituting these linear combinations into the expressions $\varphi_{u_{i}}$, we find that, for $i<n, \varphi_{u_{t}}$ is a linear combination of $\Omega_{j n}, j=1, \ldots, n$, and hence of $\varphi(h)$ and $\varphi_{i}, i<n$. This shows that all the directional derivatives of $\varphi$ at $h$ are linear combinations of $\varphi(h)$ and $n$ such directional derivatives. Hence the rank of $\varphi$ is $n$, which is what was to be proved. 
It follows from this proposition that the image of $\varphi$ is an immersed submanifold of dimension $n$ in $P^{N *}$. More precisely, let us say that $x, y \in G_{n-1} T\left(M^{n}\right)$ are equivalent if there is a curve $C$ joing $x$ and $y$ in $G_{n-1} T\left(M^{n}\right)$ such that $\varphi(C)=\varphi(x)$. The equivalence classes form a differentiable manifold $M^{*}$ and $\varphi$ induces an immersion $\varphi^{\prime}: M^{*} \rightarrow P^{N *}$. We call $\varphi^{\prime}$, or $M^{*}$ itself, the dual manifold of $f: M^{n} \rightarrow E^{N}$. Clearly the image of a fibre under $\varphi, \varphi\left(G_{n-1} T_{p}\right)$, is an algebraic variety; hence the dual manifold contains a family of algebraic varieties. We shall see later that these are in fact Veronese manifolds. But our immediate aim is to show that the dual manifold contains a family of conics.

Lemma 4.3. Let $P^{a}$ and $P^{b}$ be linear subspaces of $P^{a+b+1}$ which are in general position, i.e. which do not meet, and let $S_{t}^{a-1}$ be a one-parameter family of hyperplanes of $P^{a}$ which form a non-singular conic in the dual space $P^{a *}$. For each $t$, let $S_{t}^{a+b}$ be the linear span of $S_{t}^{a-1}$ and $P^{b}$. Then the family of hyperplanes $S_{t}^{a+b}$ forms a non-singular conic in $P^{a+b+1 *}$. If $Q$ is the hyperquadric of $P^{a}$ enveloped by $S_{t}^{a-1}$ and $Q^{\prime}$ the hyperquadric of $P^{a+b+1}$ enveloped by $S_{t}^{a+b}$, then $Q^{\prime}$ consists of linear spaces of dimension $b+1$, each of which is spanned by $P^{b}$ and a point of $Q$, and every such linear space lies in $Q^{\prime}$.

Proof. Choose homogeneous coordinates $x_{0}, \ldots, x_{a+b+1}$ in $P^{a+b+1}$ such that $P^{a}$ is defined by $x_{a+1}=\cdots=x_{a+b+1}=0, P^{b}$ is defined by $x_{0}=\cdots=x_{a}=0$, and $S_{t}^{a-1}$ consists of hyperplanes

$$
\alpha_{0} x_{0}+\cdots+\alpha_{a} x_{a}=0
$$

such that $-\alpha_{0}^{2}+\alpha_{1}^{2}+\alpha_{2}^{2}=0$ and $\alpha_{3}=\cdots=\alpha_{a}=0$. Then the family $S_{t}^{a+b}$ consists of hyperplanes

$$
\alpha_{0} x_{0}+\cdots+\alpha_{a+b+1} x_{a+b+1}=0
$$

such that $-\alpha_{0}^{2}+\alpha_{1}^{2}+\alpha_{2}^{2}=0$ and $\alpha_{3}=\cdots=\alpha_{a+b+1}=0$. But this is clearly a non-singular conic in $P^{a+b+1 *}$, which proves the first assertion of the lemma.

Now the equations of $Q$ in $P^{a+b+1}$ are

$$
-x_{0}^{2}+x_{1}^{2}+x_{2}^{2}=0, \quad x_{a+1}=\cdots=x_{a+b+1}=0 .
$$

The equation of $Q^{\prime}$ is just $-x_{0}^{2}+x_{1}^{2}+x_{2}^{2}=0$, so that $Q^{\prime}$ consists exactly of the linear spans of $P^{b}$ and points of $Q$. This completes the proof of the lemma.

Lemma 4.4. a) Let $V^{n} \subset P^{N}$ be a Veronese manifold and $V^{n-2} \subset V^{n} a$ Veronese submanifold of codimension 2 . Then the set of all e.s. hyperplanes of $V^{n}$ which contain $V^{n-2}$ is a one-parameter family $S_{t}$ which forms a nonsingular conic in the dual space $P^{N *}$. The hyperquadric enveloped by $S_{t}$ contains $V^{n}$. 
b) Suppose $n \geqq 2$ and $g: M^{n} \rightarrow E^{N}$ a non-degenerate $C^{2}$ immersion (we assume no other special properties). Let $p \in M^{n}, l$ a linear subspace of dimension $n-2$ through the origin of $T_{p}$, and $h_{t}$ the family of hyperplanes of $T_{p}$ containing $l$. Let $H_{1}$ denote the family of e.s. hyperplanes of $g$ containing the $f^{N}\left(h_{t}\right)$. Then $H_{t}$ forms a non-singular conic in $P^{N *}$. $H_{i}$ envelops a hyperquadric which contains all tangent and curvature vectors at $p$ to curves on $M^{n}$ through $p$.

Proof. a) We prove this by induction on $n$. For $n=1, V^{n}$ is a nonsingular conic and $S_{t}$ is just the family of its tangent lines. The truth of Part a) is clear. Let us assume Part a) for $n-1 \geqq 1$. Choose a point $p \in V^{n-2}$, and let $m$ denote the tangent space to $V^{n-2}$ at $p$. Then $S_{t}$ consists of the linear spans of the tangent space to $V^{n-1}$ at $p, T_{p}$, with the e.s. hyperplanes $S_{t}^{\prime}$ of $\mathscr{r}\left(S_{p}\right)$ containing $\mathscr{r}\left(m \cap S_{p}\right)$. By the induction hypothesis, $S_{f}^{\prime}$ forms a non-singular conic in the dual space of the indicatrix plane $I_{p}$. Hence by Lemma $4.3 S$, forms a non-singular conic in $P^{N *}$. Again by Lemma 4.3 the hyperquadric $Q^{\prime}$ enveloped by $S_{t}$ contains the linear spans of $T_{p}$ and the points of the hyperquadric $Q^{\prime}$ of $I_{p}$ enveloped by $S_{t}^{\prime}$. $Q^{\prime} \supset \mathscr{N}\left(S_{p}\right)$, by the induction hypothesis. Hence $Q$ contains all tangent and curvature vectors at $p$ of curves on $V^{n}$ through $p$. It follows that $Q$ contains all conics on $V^{n}$ passing through $p$. Since any point of $V^{n}$ may be joined to $p$ by such a conic, $Q$ must contain $V^{n}$. This proves Part a).

The proof of Part b) is very simple. $H_{t}$ consists of the linear spans of $T_{p}$ and the e.s. hyperplanes of $\mathscr{N}\left(S_{p}\right)$ containing $\mathscr{N}\left(l \cap S_{p}\right)$. These last form a non-singular conic in the dual space of $I_{p}$, by Part a). Hence by Lemma $4.3 H_{t}$ forms a non-singular conic in $P^{N *}$. $H_{t}$ envelops a hyperquadric which contains the linear spans of $T_{p}$ with points of $\mathscr{N}\left(S_{p}\right)$. This completes the proof.

Let us suppose $n \geqq 2$ and consider again the dual manifold $\varphi^{\prime}$ : $M^{*} \rightarrow P^{N *}$. If $H \in \varphi^{\prime}\left(M^{*}\right)$ and $H=\varphi(h)$, where $h \in G_{n-1} T_{p}$, then choose an $(n-2)$-dimensional linear subspace $h^{\prime} \subset h$ through the origin of $T_{p}$. By construction $\varphi^{\prime}\left(M^{*}\right)$ contains the linear spans of $T_{p}$ and the e.s. hyperplanes, in $I_{p}$, of $\mathscr{N}\left(S_{p}\right)$ which contain $\mathscr{N}\left(h^{\prime} \cap S_{p}\right)$. But by Lemma 4.4 b) these hyperplanes form a non-singular conic in $P^{N *}$. Hence given any point $x \in M^{*}$, there exists a closed embedded curve $C$ on $M^{*}$ passing through $x$ such that $\varphi^{\prime}(C)$ is a non-singular conic in $P^{N *}$. Such a curve we call an $s$-curve on $M^{*}$. Given $x \in M^{*}$, we call the set of all points of $M^{*}$ which can be joined to $x$ by an s-curve the wedge of $x, W_{x}$. We have just seen that $W_{x}$ contains points of $M^{*}$ other than $x$, for every $x$.

Proposition 4.5. Let $n \geqq 2$. For every $x \in M^{*}$ there is an $(n-1)$-parameter family of s-curves passing through $x . W_{x}-\{x\}$ is open in $M^{*}$. The tangent lines at $x$ to the s-curves through $x$ fill out an open set in $T_{x}$. 
We first prove the converse of Lemma 4.1 under the assumption that every hyperplane which supports to the second order supports to the third order.

Lemma 4.6. If $H \in P^{N *}$, then $\pi\left(\varphi^{-1}(H)\right)$ is an embedded submanifold of codimension one in $M^{n}$. If $\varphi(h)=H, \pi(h)=p$, then $\pi\left(\varphi^{-1}(H)\right)$ is tangent to $h$ at $p$.

Proof. Since $\varphi$ has rank $n, \varphi^{-1}(H)$ is an embedded submanifold of $G_{n-1} T\left(M^{n}\right)$ of dimension $n-1 . \varphi^{-1}(H)$ cannot have a tangent vector in common with any fibre of $G_{n-1} T\left(M^{n}\right)$, since $\varphi$ restricted to any fibre has rank $n-1$. And $\varphi^{-1}(H)$ cannot meet any fibre in more than one point, since $\varphi$ maps each fibre in a one-to-one fashion. It follows that $\pi\left(\varphi^{-1}(H)\right)$ is an embedded submanifold of dimension $n-1$ of $M^{n}$. If $p \in \pi\left(\varphi^{-1}(H)\right)$, then $H$ contains the curvature vectors to all curves on $\pi\left(\varphi^{-1}(H)\right)$ through $p$ at $p$, since $H \supset \pi\left(\varphi^{-1}(H)\right)$. It follows that $H \supset \mathscr{N}(h)$, where $h$ is the tangent space to $\pi\left(\varphi^{-1}(H)\right)$ at $p$. Hence $H=\varphi(h)$. This completes the proof.

Proof of Proposition 4.5. Let $x \in M^{*}, H=\varphi^{\prime}(x)$. Let $B$ denote the restriction of the bundle $G_{n-1} T\left(M^{n}\right)$ to $\pi\left(\varphi^{-1}(H)\right)$; that is to say, let $B=\pi^{-1}\left(\pi\left(\varphi^{-1}(H)\right)\right)$. Then by definition $\varphi^{\prime}\left(W_{x}\right)=\varphi(B)$. To show that $W_{x}-\{x\}$ is open in $M^{*}$ it suffices therefore to show that $\varphi$ has rank $n$ on $B-\varphi^{-1}(H)$.

To show this, let $y \in W_{x}, y \neq x$, be arbitrary, $L=\varphi^{\prime}(y)$. Then there is an $l \in B$ such that $\varphi(l)=L$. Let $p=\pi(l)$, and let $h \subset T_{p}$ be the tangent space to $\pi\left(\varphi^{-1}(H)\right)$ at $p$. Then $h \neq l$, since $\varphi(h)=H$ by Lemma 4.6 and $x \neq y$. Hence $h$ and $l$ intersect transversally. Choose local coordinates $u_{1}, \ldots, u_{n}$ on $M^{n}$ in a neighborhood of $p$ such that $\pi\left(\varphi^{-1}(H)\right)$ is defined in that neighborhood by $u_{n-1}=0$, (so that $h$ is spanned by $\partial / \partial u_{1}, \ldots, \partial / \partial u_{n-2}$, $\left.\partial / \partial u_{n}\right)$, and such that $l$ is spanned by $\partial / \partial u_{1}, \ldots, \partial / \partial u_{n-1}$. Let $\boldsymbol{h}_{i}(t)$ denote the linear span of

$$
\frac{\partial}{\partial u_{1}}, \ldots, \frac{\partial}{\partial u_{i-1}}, \frac{\partial}{\partial u_{i}}+t \frac{\partial}{\partial u_{n}}, \frac{\partial}{\partial u_{i+1}}, \ldots, \frac{\partial}{\partial u_{n-1}},
$$

the derivatives being evaluated at $p$. To show that $\varphi$ has rank $n$ on $B$ at $y$ it suffices to show that

$$
\varphi(h), \frac{d \varphi \circ h_{1}}{d t}(0), \ldots, \frac{d \varphi \circ h_{n-1}}{d t}(0), \frac{\partial \varphi}{\partial u_{n}}(p)
$$

are linearly independent. But these were computed and shown to be linearly independent in the course of the proof of Proposition 4.2. Hence $W_{x}-\{x\}$ is open as asserted.

In order to show that the tangent lines to the $s$-curves through $x$ fill out an open subset of $T_{x}$, we observe first that an $s$-curve through $x$ is 
constructed by choosing an $(n-2)$-plane $h^{\prime}$ through the origin of $T_{p}$, for some point $p \in \pi\left(\varphi^{-1}(H)\right)$, with $h^{\prime} \subset h$, where $h$ is such that $\varphi(h)=H$, and then taking the image under $\varphi$ of all $h \supset h^{\prime}, h \in B$. Let us call the set of all such $h^{\prime}, B^{\prime}$. So given such an $h^{\prime}$, we compute the tangent line at $h^{\prime}$ of the corresponding $s$-curve, call it $\tau$, differentiate the resulting expression along $n-1$ independent directions in $B^{\prime}$, and show that $\tau$ and these derivatives are linearly independent.

Let $h^{\prime}$ be arbitrarily chosen as above, $h^{\prime} \subset T_{p}, h \supset h^{\prime}$ so that $\varphi(h)=H$. Choose local coordinates $x_{1}, \ldots, x_{n}$ on $M^{n}$ in a neighborhood of $p$, so that $\pi\left(\varphi^{-1}(H)\right)$ is defined in that neighborhood by $x_{n}=0$ (so that $h$ is spanned by $\partial / \partial x_{1}, \ldots, \partial / \partial x_{n-1}$, by Lemma 4.6), and so that $h^{\prime}$ is spanned by $\partial / \partial x_{1}, \ldots, \partial / \partial x_{n-2}$. Now let $h_{i}^{\prime}(r)$ denote the linear span of

$$
\frac{\partial}{\partial x_{1}}, \ldots, \frac{\partial}{\partial x_{i-1}}, \frac{\partial}{\partial x_{i}}+r \frac{\partial}{\partial x_{n-1}}, \ldots, \frac{\partial}{\partial x_{n-2}},
$$

and let $h_{i}(r, t)$ denote the linear span of

$$
h_{i}^{\prime}(r) \text { and } \frac{\partial}{\partial x_{n-1}}+t \frac{\partial}{\partial x_{n}} .
$$

Note that $h_{i}^{\prime}(r) \in B^{\prime}$, and for fixed $r, h_{i}(r, t)$ is an s-curve through $x$ with $\varphi\left(h_{i}(r, 0)\right)=H$. This $s$-curve corresponds to $h_{i}^{\prime}(r)$. We call the curve $\varphi\left(h_{i}(r, t)\right)$, for fixed $r, C_{i r}$. The tangent vector to $C_{i r}$ at $H, \tau_{i r}$, is spanned by $\varphi\left(h_{i}(r, 0)\right)$ and $d / d t \varphi\left(h_{i}(r, 0)\right)$, which we proceed to compute.

From (4.1), (4.2), and using the notation of the proof of Proposition 4.2, we find that $\varphi\left(h_{i}(r, t)\right)$ must contain

and

$$
Y, Y_{1}, \ldots, Y_{n}, Y_{j k}, \quad(j \neq i, n-1, n),
$$

$$
\begin{array}{ll}
Y_{i i}+r Y_{i n-1}, & Y_{i n-1}+t Y_{i n}, \\
Y_{n-1 i}+r Y_{n-1 n-1}, & Y_{n-1 n-1}+t Y_{n-1 n}, \\
Y_{n i}+r Y_{n n-1}, & Y_{n n-1}+t Y_{n n} .
\end{array}
$$

These vectors are linearly dependent. However, with allowance made for the symmetry $X_{j k}=X_{k j}$, if we leave out $Y_{n-1 i}+r Y_{n-1 n-1}$, the remaining are linearly independent for $r, t=0$, and hence certainly for small values of $r, t$. These vectors, with the indicated one left out, are sufficient in number to span $\varphi\left(h_{i}(r, t)\right)$. If we multiply them together and calculate the derivative of the resulting expression, we obtain

$$
\frac{d \varphi \circ h_{i}}{d t}(r, 0)= \pm \Omega_{n-1 n} \pm r \Omega_{i n}
$$

We also find that

$$
H=\varphi\left(h_{i}(r, 0)\right)= \pm \Omega_{n n} .
$$

14 Inventiones math., Vol. 13 
The tangent line of $C_{i r}$ at $t=0, \tau_{i r}$, is represented by the wedge product of these last two expressions in $\Lambda^{2}\left(\Lambda^{N} R^{N+1}\right)$ :

Hence

$$
\tau_{i r}= \pm \Omega_{n n} \wedge \Omega_{n-1 n} \pm r \Omega_{n n} \wedge \Omega_{i n} .
$$

$$
\tau_{i}=\frac{d \tau_{i r}}{d r}(0)= \pm \Omega_{n n} \wedge \Omega_{i n} .
$$

Also, if $\tau$ denotes the tangent to the s-curve determined by $h^{\prime}$, we have

Hence

$$
\tau= \pm \Omega_{n n} \wedge \Omega_{n-1 n} \text {. }
$$

$$
\begin{aligned}
\frac{\partial \tau}{\partial x_{n-1}}= & \pm\left[Y \wedge Y_{1} \wedge \cdots \wedge Y_{n} \wedge(\cdots)\right] \wedge \Omega_{n-1 n} \\
& \pm \Omega_{n n} \wedge\left[Y \wedge Y_{1} \wedge \cdots \wedge Y_{n-1} \wedge Y_{n-1 n} \wedge \prod_{\substack{j \leq k \\
(j . k) \neq(n-1, n)}} Y_{j k}\right. \\
& \left.+Y \wedge Y_{1} \wedge \cdots \wedge Y_{n} \wedge(\cdots)\right] .
\end{aligned}
$$

Hence $\tau, \tau_{1}, \ldots, \tau_{n-2}, \partial \tau / \partial x_{n-1}$ are linearly independent, which shows that the tangent lines to $s$-curves at $x$ fill out an open set in $T_{x}$. This completes the proof of the proposition.

Proposition 4.7. $\varphi^{\prime}: M^{*} \rightarrow P^{N *}$ is non-degenerate.

The proof depends on the following lemma and proposition.

Lemma 4.8. Let $X: M^{n} \rightarrow P^{N}$ be a non-degenerate immersion of class $C^{2}$. Then there is no open set $U \subset M^{n}$ such that the tangent projective spaces to $X$ at all points of $U$ have a common point.

Proof. Suppose there is such an open set $U$. Let $P$ be a point common to the tangent projective spaces at the points of $U$. Choose as hyperplane at infinity in $P^{N}$ some hyperplane not containing $P$, and take $P$ to be the origin of $E^{N} \subset P^{N} . X(U)$ is not contained in the hyperplane at infinity, since $X$ is non-degenerate. Hence there is an open set $U^{\prime} \subset U$ such that $X: U^{\prime} \rightarrow E^{N}$, and the tangent spaces to $X$ at the points of $U^{\prime}$ pass through the origin.

Let $U^{\prime \prime} \subset U^{\prime}$ be the domain of some local coordinate system $x_{1}, \ldots, x_{n}$. There must exist smooth functions $\alpha_{1}, \ldots, \alpha_{n}$ such that

$$
X \equiv \alpha_{1} X_{1}+\cdots+\alpha_{n} X_{n} \quad \text { in } U^{\prime \prime}, \quad X_{i}=\frac{\partial X}{\partial x_{i}} .
$$

Differentiating this with respect to $x_{i}$, we obtain

$$
X_{i}=\frac{\partial \alpha_{1}}{\partial x_{i}} X_{1}+\cdots+\frac{\partial \alpha_{n}}{\partial x_{i}} X_{n}+\alpha_{1} X_{1 i}+\cdots+\alpha_{n} X_{n i}
$$


But since $X$ is non-degenerate, the $X_{i}$ and $X_{j k}, j \leqq k$, are linearly independent. Hence $x_{3} \equiv 0$ for $j \neq i$. But this is true for any $i$; hence $\alpha_{j} \equiv 0$ for all $j$. But this implies that $X \equiv 0$ in $U^{\prime \prime}$, a contradiction.

Proposition 4.9. Let $X: M^{n} \rightarrow P^{N}$ be a non-degenerate immersion of class $C^{2}$. Then

a) the e.s. hyperplanes of $X$ have no common point in $P^{N}$;

b) if $p \in M^{n}$, the intersection of the e. s. hyperplanes of $X$ at $p$ is $T_{p}$.

Proof. We proceed by induction. If $n=1$, we are concerned with a non-degenerate plane curve; the e.s. hyperplanes are just the tangent lines. In this case the proposition is well-known. Now assume the proposition for dimensions lower than $n$. Since the proposition is of a local and projective nature, we may assume $X: M^{n} \rightarrow E^{N}$ and apply our metric constructions. Consider the e.s. hyperplanes at $p$. Their intersection certainly contains $T_{p}$. If it is larger than $T_{p}$ it meets the indicatrix plane $I_{p}$, and hence the e.s. hyperplanes of $\mathscr{N}\left(S_{p}\right)$ have a common point. But this is impossible by the induction hypothesis. This proves Part $b$ ).

To prove Part a) we observe that, by Part b), if all the e.s. hyperplanes of $X$ have a common point, then all the tangent spaces of $X$ have a common point. But this is impossible, by Lemma 4.8. This concludes the proof.

By Proposition 4.9 b), the condition that any hyperplane which supports to the second order supports to the third order may be put in the following form: if $p \in M^{n}, x(t)$ a curve on $M^{n}$ through $p$ with unit tangent vector $v$ at $p$ such that $x(0)=p$, then $X^{\prime \prime \prime}(0)$ lies in the linear span of $T_{p}$ and the tangent space to $\mathscr{N}$ at $v$, where $X(t)=f(x(t))$.

Proof of Proposition 4.7. Suppose $\varphi^{\prime}$ is degenerate at $x \in M^{*}$. Then there is a hyperplane $J \subset P^{* *}$ containing all first and second derivatives of curves on $M^{*}$ at $x . J$ must then contain the images of all $s$-curves through $x$. Hence, by Proposition 4.5, $J$ contains the image under $\varphi$ of an open subset $U$ of $G_{n-1} T\left(M^{*}\right)$. Assume $U$ is the largest such open set. Now if $U$ meets a fibre $F$, it meets $F$ in an open set of $F$, and hence $F \subset U$, since $\varphi$ restricted to $F$ is real algebraic. It follows that all the e.s. hyperplanes of $f$ at the points of $\pi(U)$, when viewed in $P^{N *}$, lie in $J$. Dually, this says that all the e.s. hyperplanes of $f$ at the points of $\pi(U)$ have a common point in $E^{N}$. But this is impossible by Proposition 4.9 a). This completes the proof.

\section{Submanifolds of $E^{N}$ Containing Many Plane Curves}

In this $\S$ we prove the following.

Theorem III. Let $M$ be a connected differentiable manifold of dimension $n>1$ and $f: M \rightarrow E^{N}, N=\frac{1}{2} n(n+3)$, a non-degenerate immersion dif- 
ferentiable of class $C^{2}$. Suppose $M$ contains a family of $C^{2}$ curves, which we call s-curves, such that each s-curve is mapped by $f$ onto a plane curve in $E^{N}$. For each $x \in M$ let $W_{x}$ denote the set of points of $M$ which can be joined to $x$ by s-curves. Suppose that $W_{x}-\{x\}$ is non-empty and open for each $x$ and that the tangent lines to the s-curves at $x$ fill out an open subset of the tangent space at $x$. Then $f(M)$ is contained in a Veronese n-manifold in $P^{N}$.

Before beginning the proof, we make a few remarks. By Proposition 4.5 the dual manifold of the last $\S$ satisfies the hypotheses of the theorem. Let us note that the hypotheses imply that if $y \in W_{x}$, then $x \in W_{y}$; and $W_{x}$ has the property that if $U$ is any neighborhood of $x$ in $M$, then $U \cap W_{x}$ has a non-empty interior.

Proof of Theorem III. Let $x \in M$ and let $j$ be a linear subspace of dimension $n-2$ of $T_{x}$. By Lemma $4.4 \mathrm{~b}$ ) the e.s. hyperplanes of $f$ at $x$ which contain $\mathscr{V}(j)$ envelop a hyperquadric $Q$ which contains the tangent and curvature vectors of all curves at $x$, in particular the $s$-curves through $x$. But the plane of an $s$-curve is spanned by the tangent and curvature vector at any point, because $f$ is non-degenerate and therefore contains no curves with curvature vanishing anywhere. It follows that $Q$ contains all $s$-curves on $M$ through $x$ and hence $Q \supset W_{x}$. In order to prove the theorem we will show that any point of $M$ has a neighborhood whose image under $f$ lies in a number of such hyperquadrics, which can be chosen in such a way that their intersection is a Veronese surface.

Let $y \in M$ be an arbitrary point. We work in a neighborhood of $y$ which is embedded by $f$. We clain that there is some point $y_{1} \in W_{y}$ such that $T_{y} \cap T_{y_{1}}$ is a linear space of dimension $<n-1$ in $E^{N}$. For if not, let $\pi: E^{N} \rightarrow N_{y}$ denote orthogonal projection into the normal space at $y$. Then the rank of $\pi$ on $f\left(W_{y}\right)$ must be $\leqq 1$, so that $\pi \circ f\left(W_{y}\right)$ is a curve, possibly with singularities. Now if $C$ is any $s$-curve on $M$ through $y, \pi f(C)$ is a segment of a generating ray of the curvature cone $\mathscr{N}\left(T_{y}\right)$. Hence if $q \in \pi f\left(W_{x}\right)$, then the line segment joining $q$ to $y$ must also lie in $\pi f\left(W_{x}\right)$. Hence since $\pi f\left(W_{x}\right)$ is a curve, it must lie in a line. But this implies that $f\left(W_{x}\right)$ lies in the linear span of that line with $T_{y}$, contradicting the non-degeneracy of $f$. Let us note here that $\pi\left(W_{y}\right) \subset \mathcal{N}\left(T_{y}\right)$.

So let us choose $y_{1} \in W_{y}$ such that $T_{y} \cap T_{y_{1}}$ has dimension less than $n-1$. Next we choose $y_{2} \in W_{y} \cap W_{v_{1}}, y_{2} \neq y$, in such a way that $T_{y_{2}} \cap T_{v_{1}}$ has dimension $<n-1$ and such that $y_{2} \notin T_{v 1}$ and $y_{1} \notin T_{y_{2}}$. This is possible since $W_{y_{1}} \cap W_{y_{2}}$ has a non-empty interior containing points arbitrarily close to $y$. We now choose $y_{3}, \ldots, y_{n+1} \in M$, by a recursive process. We assume that $y_{1}, \ldots, y_{i}, i<n+1$, have been chosen in such a way that

$$
\left.1_{i}\right) y, y_{1}, \ldots, y_{i} \in W_{y} \cap W_{y_{1}} \cap \cdots \cap W_{y_{i}}, f\left(y_{j}\right) \neq f(y) \text {, all } j \leqq i \text {; }
$$


$2_{i}$ ) if $\pi_{j}$ denotes the projection into the normal space of $f$ at $y_{j}$, then there exist $z_{j 1}, \ldots, z_{j i} \in T_{y_{j}}$ which are in general position in $T_{y_{j}}$ and such that $\mathscr{N}\left(z_{j k}\right)=\pi_{j} f\left(y_{k}\right)$, for all $k \leqq i$;

$\left.3_{i}\right) T_{y_{1}} \cap \cdots \cap T_{y_{i}}$ has dimension $\leqq n-i$.

(Let us note that for $i=2$, the set $\left\{y_{1}, y_{2}\right\}$ already chosen satisfies conditions $\left.\left.\left.1_{i}\right), 2_{i}\right), 3_{i}\right)$.) Let us now consider the interior, $U$, of $W_{y} \cap W_{y_{1}} \cap \cdots \cap W_{y_{2}}$. If every point $x$ of $U$ has the property that $T_{x} \supset T_{y_{1}} \cap \cdots \cap T_{y_{1}}$, then the assumed non-degeneracy and Lemma 4.8 give a contradiction. Hence there is some open set $U^{\prime} \subset U$ with the property that if $x \in U^{\prime}$, then $T_{x} \cap T_{y_{1}} \cap \cdots \cap T_{y_{i}}$ has dimension $\leqq n-i-1$. Now let $h_{j}$ denote the linear span, in $T_{y_{j}}$, of $z_{j 1}, \ldots, z_{j i}$. Let $P_{j}$ denote the linear span of $T_{y_{j}}$ and $\mathscr{N}\left(h_{j} \cap S_{y_{j}}\right)$. Since $\mathcal{N}\left(h_{j} \cap S_{y_{j}}\right)$ lies in a linear space of dimension $\frac{1}{2}(i-2)(i+1), P_{j}$ must have dimension $<N$. Hence, by the non-degeneracy, $P_{j}$ can contain no open set of $M$. It follows that $U^{\prime \prime}=$ $U^{\prime}-\bigcup P_{j}$ is non-empty, and so we choose $y_{i+1} \in U^{\prime \prime}$. Conditions $1_{i+1}$ ), $2_{i+1}$ ), and $3_{i+1}$ ) are now easily verified, with the exception of $2_{i+1}$ ) for the case $j=i+1$.

But suppose $z_{i+11}, \ldots, z_{i+1 i+1}$ are so chosen that $\mathscr{N}\left(z_{i+1 k}\right)=\pi_{i+1} f\left(y_{k}\right)$. If these $z$ 's are not in general position, then one of them lies in the linear span of the others, say $z_{i+11}$ lies in the linear span of $z_{i+12}, \ldots, z_{i+1 i+1}$. Call this linear span $Q$. Let $Q^{\prime}$ denote the linear span of $T_{y_{1+1}}$ and $\mathscr{r}\left(Q \cap S_{y_{i+1}}\right)$. Then $Q^{\prime}$ contains $f\left(y_{1}\right), \ldots, f\left(y_{i+1}\right)$. But $Q^{\prime}$ cannot contain an open set of $M$. And everything established so far remains true if we vary $y_{1}$ slightly. So vary $y_{1}$ slightly, to get it outside of $Q^{\prime}$. And repeat this argument until $z_{i+11}, \ldots, z_{i+1 i+1}$ are in general position. This completes our account of the construction of $y_{1}, \ldots, y_{n+1}$. Note that $T_{y_{1}} \cap \cdots \cap T_{y_{n+1}}$ is empty.

Suppose we have an e.s. hyperplane $H$ of $f$ at $y_{j}$ and suppose $H$ contains $f\left(y_{k}\right)$. We claim that $H$ is also an e.s. hyperplane of $f$ at $y_{k}$. To show this, we observe first of all that since $H$ supports $\mathscr{N}\left(T_{y}\right)$ it supports any $s$-curve through $y_{j}$, and hence it supports $W_{y}$. Hence it supports $\mathscr{N}\left(T_{y_{k}}\right)$ and contains $T_{y_{k}}$. Let $C$ be an $s$-curve joining $y_{j}$ and $y_{k} \cdot \pi_{j} f(C)$ is a segment lying on the curvature cone $\mathscr{N}\left(T_{y_{j}}\right)$, and this segment is contained in $\mathscr{N}(l)$, where $l$ is the tangent line to $C$ at $y_{j}$. Since $H$ contains $T_{y_{j}}$ and $f\left(y_{k}\right), H$ contains $f(C)$ and hence it contains this segment. Now by hypothesis the tangent lines to the $s$-curves through $y_{j}$ fill out an open set in $T_{y_{j}}$. Since $H \cap \mathscr{N}\left(S_{y_{j}}\right)$ is, by definition, a Veronese submanifold of dimension $(n-2)$ of $\mathscr{N}\left(S_{y_{j}}\right), H$ must contain an $(n-2)$-parameter family of $s$-curves containing $C$. This family sweeps out an $(n-1)$ dimensional submanifold of $M$ containing $y_{k}$, and hence, by Lemma 4.1, $H$ is an e.s. hyperplane at $y_{k}$, which establishes the claim.

Let $i \leqq n+1$, and pick $j \leqq n+1, j \neq i$. Let $h_{j i}$ denote the linear span of $z_{j 1}, \ldots, z_{j i-1}, z_{j i+1}, \ldots, z_{j n+1}$. Let $H_{i}$ be the e.s. hyperplane at $y_{i}$ con- 
taining $\mathscr{N}\left(h_{j i}\right)$. Note that $H_{i}$ contains $f\left(y_{k}\right)$ for all $k \neq i$ and is an e.s. hyperplane at each of them. Now for each $i, j \leqq n+1, i \neq j$, choose $k \neq i, j$, $k \leqq n+1$, and let $h_{k i j}$ denote the linear span of $\left\{z_{k 1}, \ldots, z_{k n+1}\right\}-\left\{z_{k i}, z_{k i}\right\}$. Consider the set $\Pi_{i j}$ of e.s. hyperplanes at $y_{k}$ containing $h_{k i j}$. By property $\left.2_{n+1}\right)$ above, and Lemma $4.4, \Pi_{i j}$ forms a non-singular conic in $P^{N *}$ and envelops a hyperquadric containing $W_{y_{k}}$, and hence a neighborhood of $y$. The hyperplanes of $\Pi_{i j}$ are e.s. hyperplanes at each $y_{k}, k \neq i, j . \Pi_{i j}$ and $\Pi_{i i}$ have $H_{i}$ in common.

Now let $k \leqq n+1$ and consider the intersection of all hyperplanes of the families $\Pi_{i j}$ such that $i, j \neq k$. We claim that this intersection is just $T_{y_{k}}$. This follows from the fact that if $w_{1}, \ldots, w_{n}$ are $n$ points, lying on a Veronese manifold $V^{n-1}$, which are not contained in a proper Veronese submanifold, and if $\Pi_{i j}^{\prime}$ denotes the family of e.s. hyperplanes of $V^{n-1}$ which contain $\left\{w_{1}, \ldots, w_{n}\right\}-\left\{w_{i}, w_{j}\right\}$, then the intersection of all the hyperplanes of the families $\Pi_{i j}^{\prime}$ is void. This, in turn, is proved by induction on $n-1$, using an argument similar to that of Proposition 4.9. The essential step in the induction is the observation that if $V^{2}$ is a Veronese manifold of dimension $i$ and $x_{1}, \ldots, x_{i+1}$ are points on $V^{i}$ not lying in a proper Veronese submanifold, then the tangent spaces to $V^{i}$ at $x_{1}, \ldots, x_{i+1}$ have no common point. This, in turn, follows from the calculation near the end of $\S 2$. Finally, since $T_{v_{1}} \cap \cdots \cap T_{y_{n+1}}=0$, there is no point common to all the hyperplanes of the families $\Pi_{i j}$.

Now draw the tangents to the conic $\Pi_{i j}$ at $H_{i}$ and $H_{j}$. They meet in a point $H_{i j}$, and the linear span of all the points $H_{i}, H_{i j}$ is the same as the linear span of all the conics $\Pi_{i j}$. This has dimension $\leqq n+\frac{1}{2} n(n+1)=N$. If it is strictly less than $N$, then all the $\Pi_{i j}$ lie in a hyperplane of $P^{N *}$, which says that all the hyperplanes of the families $\Pi_{i j}$ have a common point. Since this is impossible, the dimension above must be $N$, which says that the set of points $H_{k}, H_{i j}, i<j$, is in general position.

Let us introduce homogeneous coordinates in $P^{N *}$ such that the coordinates of $H_{k}$ or $H_{i j}$ are all zero, but for one. For convenience we call these coordinates $\xi_{i}^{*}, \xi_{j k}^{*}, 1 \leqq i \leqq n+1,1 \leqq j<k \leqq n+1$, and we require that

$$
\begin{array}{lll}
H_{i} \text { is the point } \xi_{i}^{*}=\delta_{u l}, & \xi_{j k}^{*}=0, & \text { all } j, k ; \\
H_{j k} \text { is the point } \xi_{m p}^{*}=\delta_{j m} \delta_{k p}, & \xi_{i}^{*}=0, & \text { all } i .
\end{array}
$$

In these coordinates $\Pi_{i j}$ has equations

$$
a_{i j} \xi_{i}^{*} \xi_{j}^{*}+b_{i j} \xi_{i j}^{* 2}=0, \quad \xi_{l}^{*}=0, \quad l \neq i, j, \quad \xi_{m p}^{*}=0, \quad(m, p) \neq(i, j),
$$

where $i<j$ and $a_{i j}$ and $b_{i j}$ are non-zero. We still have the freedom to normalize the coordinates, so we may incorporate the constants $a_{i j}, b_{i j}$ in $\xi_{i j}^{*}$. The first equation becomes then

$$
\varepsilon_{i j} \xi_{i}^{*} \xi_{j}^{*}-\xi_{i j}^{* 2}=0, \quad \varepsilon_{i j}= \pm 1 .
$$


If $\xi_{i}, \xi_{j k}$ are the homogeneous coordinates in $P^{N}$ dual to the $\zeta_{i}^{*}, \xi_{j k}^{*}$, then the hyperquadric enveloped by $\Pi_{i j}$ has equation

$$
\varepsilon_{i j} \xi_{i} \xi_{j}-\frac{1}{4} \xi_{i j}^{2}=0 \text {. }
$$

Now each of these hyperquadrics, and hence the intersection of all of them, contains a neighborhood of $y$ in $M$. In this neighborhood there must be a point whose first $n+1$ coordinates, $\xi_{1}, \ldots, \xi_{n+1}$, are all different from zero. For otherwise this neighborhood would be contained in a finite union of hyperplanes, contradicting the non-degeneracy of $f$. By reversing the signs of the $\xi_{i}$, if necessary, we can arrange that $\xi_{1}, \ldots, \xi_{n+1}$ are all positive at some point in this neighborhood. We must then have all $\varepsilon_{i j}=1$. Our equations thus become

$$
\xi_{i} \xi_{j}-\frac{1}{4} \xi_{i j}^{2}=0 \text {. }
$$

In order to solve these equations, we set $x_{i}=\left|\xi_{i}\right|^{\frac{1}{2}}$. Then $\xi_{i j}^{2}=4\left|\xi_{i}\right|\left|\xi_{j}\right|$, so

$$
\xi_{i}= \pm x_{i}^{2}, \quad \xi_{i j}= \pm 2 x_{i} x_{j} .
$$

This is a parametric representation of the various pieces of the intersection of our hyperquadrics, for various choices of the signs. But given any of these pieces, we can reverse the signs of $\xi_{i j}$ and $\xi_{i}$ as necessary to get it into the form

$$
\xi_{i}=x_{i}^{2}, \quad \xi_{i j}=2 x_{i} x_{j}
$$

But this we recognize as our parametrization of a Veronese manifold. Hence there is some neighborhood $U$ of $y$ in $M$ such that $f(U)$ lies in a Veronese manifold.

Now $y \in M$ is an arbitrary point. Since $M$ is connected, by analytic continuation we conclude that $f(M)$ lies on a Veronese $n$-manifold. This concludes the proof of Theorem III.

\section{Proof of Theorems I and II}

We prove Theorem II as follows. Let $f: M^{n} \rightarrow E^{N}$ be a non-degenerate immersion of class $C^{4}$ which has the property that any hyperplane which supports to the second order supports to the third. We may construct the dual manifold $\varphi^{\prime}: M^{*} \rightarrow P^{N *}$, which is differentiable of class $C^{2}$ and which, by Propositions 4.5 and 4.7 , satisfies the hypotheses of Theorem III. Hence, by Theorem III, $\varphi^{\prime}\left(M^{*}\right)$ is contained in a Veronese $n$ manifold $V^{*}$.

Now let $V^{\prime}$ be a Veronese manifold of dimension $n$ lying in $P^{N}$. Since it has the two-piece property, by Corollary 3.4 it has the property that any hyperplane which supports to the second order supports to the third. Also $V^{\prime}$ is non-degenerate. Hence by the last paragraph its dual manifold $V^{*} \rightarrow P^{N *}$ lies on a Veronese manifold. This last map is one- 
to-one by $\S 2$. And it is onto the Veronese manifold, because $V^{\prime}$ is compact and any Veronese manifold is connected. Now apply a projective transformation of $P^{N *}$ which brings $V^{\prime *}$ to $V^{*}$. Since the $\varphi$-mapping for $V^{\prime}$ may be constructed in a projectively invariant fashion, the adjoint projective transformation of $P^{N}$ brings $V^{\prime}$ to a Veronese manifold $V$ whose dual manifold is $V^{*}$.

Consider a point $p$ of $M^{n}$ or $V$, and consider the e.s. hyperplanes at $p$, $\varphi\left(G_{n-1} T_{p}\right)$. We claim that they form a Veronese $(n-1)$-manifold in $P^{N *}$. For if we take homogeneous coordinates $\xi_{1}, \ldots, \xi_{N+1}$ in $P^{N}$ such that $T_{p}$ is defined by $\xi_{n+2}=\cdots=\xi_{N+1}=0$ and $I_{p}$, the indicatrix plane, by $\xi_{1}=\cdots=\zeta_{n+1}=0$, then if

$$
\sum_{i=n+2}^{N+1} \alpha_{i} \xi_{i}=0
$$

is an e.s. hyperplane of $\mathcal{N}\left(S_{p}\right)$, the $\alpha_{i}$ must satisfy some system of equations defining a Veronese $(n-1)$-manifold, since by the last paragraph the e.s. hyperplanes of the Veronese manifold $\mathscr{N}\left(S_{p}\right)$ form a Veronese $(n-1)$ manifold. Now the e.s. hyperplanes $\varphi\left(G_{n-1} T_{p}\right)$ are just the linear spans of $T_{p}$ and the e.s. hyperplanes of $\mathscr{N}\left(S_{p}\right)$. Hence these are hyperplanes

$$
\sum_{i=1}^{N+1} \alpha_{i} \xi_{i}=0
$$

such that $\alpha_{1}=\cdots=\alpha_{n+1}=0$, and $\alpha_{n+2}, \ldots, \alpha_{N+1}$ satisfy the equations mentioned above. But these together are the equations of a Veronese $(n-1)$-manifold lying in $P^{N *}$, which proves the claim.

Now to each point $p \in M^{n}$ we can assign the Veronese $(n-1)$-manifold $\Phi_{M}(p)=\varphi\left(G_{n-1} T_{p}\right)$, and to each point $q \in V$ the Veronese $(n-1)$-manifold $\Phi_{V}(q)=\varphi\left(G_{n-1} T_{q}\right)$. The range space of $\Phi_{M}$ and $\Phi_{V}$ is the set of all Veronese $(n-1)$-submanifolds of $V^{*}$, which may be identified with a projective space of dimension $n, P^{n}$. Now $\Phi_{V}$ must be onto and have constant rank, because $V$ is equivariantly embedded and the mapping $\varphi$ is projectively invariant. It follows that $\Phi_{V}$ is also one-to-one, since $V^{V}$ and $P^{n}$ are homeomorphic and the fundamental group is $Z_{2}$.

Consider now the mapping $\Phi_{V}^{-1} \Phi_{M}: M^{n} \rightarrow V$. It is differentiable, by construction. If $\Phi_{V}^{-1} \Phi_{M}(p)=q$, then by Proposition $4.9 T_{p}=T_{q}$. If we represent $f: M \rightarrow E^{N}$ as a position vector function $X$, and $V$ by a position vector function $Y$, then we can write

$$
Y \circ \Phi_{V}^{-1} \Phi_{M}=X+\alpha_{1} X_{1}+\cdots+\alpha_{n} X_{n},
$$

where $x_{1}, \ldots, x_{n}$ are local coordinates on $M^{n}$ and $X_{i}=\partial / \partial x_{i} X$. Differentiating, we obtain

$$
\left(Y \circ \Phi_{V}^{-1} \Phi_{M}\right)_{i}=X_{i}+\sum \frac{\partial \alpha_{i}}{\partial x_{i}} X_{j}+\sum \alpha_{j} X_{i j}
$$


But since $T_{p}=T_{q}$, and $X_{i j}, X_{i}$ are linearly independent, we must have $\alpha_{j}=0,1 \leqq j \leqq n$. But this implies that $f\left(M^{n}\right) \subset V$. This completes the proof of Theorem II.

Finally we prove Theorem I. Let $M^{n}$ be compact and let $f: M^{n} \rightarrow E^{N}$ be a $C^{4}$ immersion which is substantial and which has the two-piece property. It is trivial to show that $M^{n}$ must be connected. Consider the set of extreme points of $f$. By Proposition 3.2 this set is non-empty and open. Let $M_{0}$ be one of its connected components. Then by Corollaries 3.7 and 3.4, $f$ restricted to $M_{0}$ is non-degenerate and has the property that any hyperplane which supports to the second order supports to the third order. Hence, by Theorem II, $f\left(M_{0}\right)$ lies on a Veronese $n$-manifold $V \subset P^{N}$. Now if $M_{0}$ had a boundary point $p$, then $f$ would be degenerate at $p$, by Proposition 3.8. By continuity, $p \in V$ and $V$ would be degenerate at $p$. But this is impossible. Hence $M_{0}$ has no boundary points, so $M_{0}=M^{n}$, and $f\left(M^{n}\right)=V$. Finally, $f$ must be an embedding, by Proposition 3.2. This completes the proof.

\section{Alternative Proofs}

We think it might be worthwhile to indicate some other proofs of Theorem I.

1) The original proof of Little is essentially that of the present paper, up to Corollary 3.4. The chief difference between his paper and the present one lies in the proof of Theorem II, which he carried out by using the given conditions on the third derivatives to introduce local coordinates on the submanifold in which the second derivatives of the immersion map vanish identically. This proof is in some sense more straightforward than that presented here, but the calculations involved are very complicated. The deduction of Theorem I from Theorem II is essentially that given in the present paper.

2) A simplified proof of Theorem I, but one which does not yield Theorem II, may be given as follows. Proposition 3.3 may be interpreted as saying that if we project $f\left(M^{n}\right)$ orthogonally into the normal space at an extreme point $p$, then the image will lie inside the convex hull of $\mathscr{N}\left(T_{p}\right)$. On the other hand, using the map $\varphi$, it may be seen that each e.s. hyperplane at $p$ meets $M^{n}$ in a submanifold of codimension one through $p$, and it can be shown that these submanifolds fill up a neighborhood of $p$. But when an e.s. of $f$ at $p$ is projected into the normal space at $p$, it goes onto an e.s. hyperplane of $\mathcal{N}\left(S_{p}\right)$. It follows that the orthogonal projection of some neighborhood of $p$ must lie on $\mathscr{N}\left(T_{p}\right)$. We can now modify the argument of $\S 5$ to prove that some neighborhood of $p$ is a Veronese manifold, and then use the concluding arguments of $\S 6$ to obtain Theorem I. This approach avoids considering the dual 
manifolds as such, and moreover requires only $C^{3}$ differentiability for $f$. But because of other applications of Theorem II we have gone the present route.

3) Kuiper's proof [4] of Theorem I for surfaces uses Morse theory to show that a tight surface in $E^{5}$ carries a two-parameter family of ellipses. It then appeals, in effect, to what we have proved here as Theorem III, which was proved for surfaces by Segre $[10,11]$.

4) An heuristic proof of Theorem I for surfaces may be given in the following way. By an argument essentially due to Banchoff [1], any e.s. hyperplane at an extreme point $p$ of an immersed surface in $E^{5}$ having the two-piece property must meet the surface in more than one point. Let us accept that it is a curve. This curve is a top set in the sense of Kuiper [4], so it must be a plane convex curve. Accepting that such curves fill out a neighborhood of $p$, it follows that orthogonal projection into the normal space at $p$ sends this neighborhood into $\mathscr{N}\left(T_{p}\right)$. We now proceed as in $\S 5$.

\section{References}

1. Banchoff,T.F.: The spherical two-piece property and tight surfaces in spheres. J. Diff. Geom. 4, 193-205 (1970).

2. Bertini, E.: Einführung in die projektive Geometrie mehrdimensionaler Räume. Vienna: L.W. Seidel und Sohn, 1924.

3. Chern, S.S., Lashof, R.K.: On the total curvature of immersed manifolds, I. Amer. Journal of Math. 78, 306-313 (1957).

4. Kuiper, N.H.: On convex maps. Nieuw Archief voor Wisk. 10, 147-164 (1962).

5. - Der Satz von Gauss-Bonnet für Abbildungen im $E^{N}$. Jahr.-Ber. DMV Bd. 69, 77-88 (1967).

6. - Minimal total absolute curvature for immersions. Inventiones Math. 10, 209-238 (1970).

7. Lane, E. P.: Projective differential geometry of curves and surfaces. Chicago: University of Chicago Press, 1932.

8. Little, J.A : On singularities of submanifolds of higher dimensional Euclidean spaces. Annali di Mat. pura ed appl. (IV) 83, 261-336 (1969).

9. Morse, $M$.: The existence of polar non-degenerate functions on differentiable manifolds. Ann. of Math. 71, 352-383 (1960).

10. Segre, C.: Su un classe di superficie degl' iperspazi legata colle equazioni lineari alle derivate parziali di $2^{\circ}$ ordine. Torino Atti 42, 1047 (1907).

11. - Le linee principali di una superficie di $S_{5}$ e une proprietà caratteristica della superficie di Veronese. Rom. Acc. L. Rend. (5) 30, 200 and 227 (1921).

\section{J.A. Little}

Department of Mathematics

University of Michigan

Ann Arbor, Michigan 48104

USA

\author{
W. F. Poh1 \\ Department of Mathematics \\ University of Minnesota \\ Minneapolis, Minn. 55455 \\ USA
}

